\section{Supratentorial lesions contribute to trigeminal neuralgia in multiple sclerosis}

Cephalalgia

2018, Vol. 38(7) 1326-1334

(C) International Headache Society 2017 Reprints and permissions: sagepub.co.uk/journalsPermissions.nav DOI: I0.II77/0333I024I7737788 journals.sagepub.com/home/cep

@SAGE

\author{
Kilian Fröhlich ${ }^{1} *$, Klemens Winder ${ }^{1} *$, Ralf A Linker', \\ Tobias Engelhorn' ${ }^{2}$, Arnd Dörfler ${ }^{2}$, De-Hyung Lee', \\ Max J Hilz ${ }^{1,3}$, Stefan Schwab' and Frank Seifert'
}

\begin{abstract}
Background: It has been proposed that multiple sclerosis lesions afflicting the pontine trigeminal afferents contribute to trigeminal neuralgia in multiple sclerosis. So far, there are no imaging studies that have evaluated interactions between supratentorial lesions and trigeminal neuralgia in multiple sclerosis patients.

Methods: We conducted a retrospective study and sought multiple sclerosis patients with trigeminal neuralgia and controls in a local database. Multiple sclerosis lesions were manually outlined and transformed into stereotaxic space. We determined the lesion overlap and performed a voxel-wise subtraction analysis. Secondly, we conducted a voxel-wise non-parametric analysis using the Liebermeister test.

Results: From 12,210 multiple sclerosis patient records screened, we identified 4I patients with trigeminal neuralgia. The voxel-wise subtraction analysis yielded associations between trigeminal neuralgia and multiple sclerosis lesions in the pontine trigeminal afferents, as well as larger supratentorial lesion clusters in the contralateral insula and hippocampus. The non-parametric statistical analysis using the Liebermeister test yielded similar areas to be associated with multiple sclerosis-related trigeminal neuralgia.

Conclusions: Our study confirms previous data on associations between multiple sclerosis-related trigeminal neuralgia and pontine lesions, and showed for the first time an association with lesions in the insular region, a region involved in pain processing and endogenous pain modulation.
\end{abstract}

\title{
Keywords
}

Voxel-based lesion symptom mapping, multiple sclerosis, trigeminal neuralgia, facial pain

Date received: 4 June 2017; revised: 2 August 2017; accepted: 19 August 2017

\section{Abbreviations}

$\begin{aligned} \text { VLSM } & \text { Voxel-based lesion symptom mapping } \\ \text { TN } & \text { Trigeminal neuralgia } \\ \text { MNI } & \text { Montreal Neurological Institute } \\ \text { FWE } & \text { Family-wise error } \\ \text { EDSS } & \text { Expanded Disability Status Scale }\end{aligned}$

\section{Introduction}

Trigeminal neuralgia (TN) affects $1-6.3 \%$ of patients with multiple sclerosis (1). Compared to the general population, multiple sclerosis patients have a highly increased, about 20-fold risk of developing TN (2). According to the International Classification of Headache Disorders of the International Headache Society (IHS) (3), TN can be distinguished in a classic form most often caused by a neurovascular contact and a symptomatic form like multiple sclerosis-related $\mathrm{TN}(3)$.

The multiple sclerosis pathology has a predilection for the optic nerves and in the brain for the periventricular white matter, including the callosal radiation

\footnotetext{
'Department of Neurology, University Hospital Erlangen, FriedrichAlexander-University Erlangen-Nürnberg, Germany

${ }^{2}$ Department of Neuroradiology, University Hospital Erlangen, FriedrichAlexander-University Erlangen-Nürnberg, Germany

${ }^{3}$ Autonomic Unit, University College London, Institute of Neurology and National Hospital for Neurology and Neurosurgery, Queen Square, London, UK

*These authors contributed equally to this work
}

\section{Corresponding author:}

Kilian Fröhlich, Department of Neurology, University Hospital Erlangen Friedrich-Alexander-University Erlangen-Nürnberg (FAU),

Schwabachanlage 691054 Erlangen, Germany.

Email: kilian.froehlich@uk-erlangen.de 
and juxtacortical U-fibers. Multiple sclerosis lesions are also found in the brainstem and spinal cord $(4,5)$. Because TN is far more frequent in multiple sclerosis patients than in the general population, $\mathrm{TN}$ might be associated with the multiple sclerosis-related pathology or specific multiple sclerosis lesion locations in the brain $(2,6-9)$. Yet, the relationship between $\mathrm{TN}$ in multiple sclerosis patients and multiple sclerosis-associated lesion sites is discussed controversially $(2,6-10)$. A few studies have shown that multiple sclerosis lesions in brain stem areas are not associated with sensory trigeminal symptoms, including paresthesia and pain (10). In contrast, other studies have linked facial sensory symptoms or symptomatic TN in multiple sclerosis patients to inflammatory lesions of the brainstem (2,6-9). However, these previous studies had the a priori hypothesis that multiple lesions in the brainstem are the primary cause of multiple sclerosis-related TN $(2,6-10)$. Lesion sites in other brain areas than the brainstem that contribute to integrating pain perception were not assessed in these studies $(6,9,11)$.

We hypothesized that multiple sclerosis lesions in other brain areas than the brainstem that are involved in pain processing may contribute to the genesis of multiple sclerosis-related TN. Therefore, we performed a retrospective analysis of collected clinical data of multiple sclerosis patients with multiple sclerosis-related $\mathrm{TN}$ and compared their clinical data and lesion pattern to a matched sample of multiple sclerosis patients without TN. To determine associations between occurrence of multiple sclerosis-related TN and multiple sclerosis lesion location, we applied a voxel-based lesion symptom mapping (VLSM) analysis (12 -16).

\section{Materials and methods}

\section{Patients}

This study was a retrospective analysis of multiple sclerosis patients seen between 2002 and 2015 by qualified neurologists at the Department of Neurology of the University Hospital Erlangen of the FriedrichAlexander-University Erlangen-Nürnberg (Erlangen, Germany). Medical reports obtained after medical visits of multiple sclerosis patients are stored in a computerized database. All patients gave written informed consent to the use of their data. In this database, we performed a search for patients with multiple sclerosis and documented persistent or a past episode of TN by using the keywords "multiple sclerosis" and "trigeminal neuralgia". We studied patients in the database who fulfilled the following inclusion criteria: (a) patients with relapsing-remitting, secondary progressive or primary progressive multiple sclerosis; (b) aged 18-75 years, and (c) with available medical reports containing details of the medical history with particular emphasis on physical examination, disease course, disease duration and first manifestation of TN. Because, in some patients, pain paroxysms cross the midline and patients experience bilateral pain, definition of lateralization was applied to the dominant side of pain. We excluded patients with the following conditions: (a) imaging or MRI sequences not available or poor imaging quality, (b) structural cerebral diseases other than multiple sclerosis, (c) evidence of chronic or persistent facial pain. Additionally, we decided in favor of an exclusion of (d) patients with $\mathrm{TN}$ and an evident vascular contact of the trigeminal nerve to avoid a possible bias and interference in our results. Vascular contact was defined as a neurovascular contact in general, irrespective of morphological changes.

For diagnosis of TN, we applied the current guidelines of the International Headache Society (IHS) at the time of the visit (3). This guideline defines $\mathrm{TN}$ as sudden, usually unilateral, severe, brief, stabbing and recurrent episodes of pain in the distribution of branches of the trigeminal nerve (3).

Diagnosis of relapsing-remitting multiple sclerosis was defined according to the current version of the McDonald criteria (17). The degree of physical disability was determined using the Expanded Disability Status Scale (EDSS) score (18).

To compare the imaging characteristics of patients with and without multiple sclerosis-related TN, we established a control group of multiple sclerosis patients in the database without documented TN. We matched the control patients for gender, age, and EDSS score (variability of 1 year in age or 0.5 points in EDSS score was tolerated, if no exact match was available). Apart from these criteria, patients in the control group were randomly selected.

\section{$M R I$ examination}

All patients underwent 1.5 or 3 Tesla (T) MRI of the brain. MRI scans included axial T1-weighted and axial T2-weighted sequences with a slice thickness of $5 \mathrm{~mm}$ for detection of infratentorial multiple sclerosis- lesion load. Axial or 3D fluid-attenuated inversion recovery (FLAIR) sequences were obtained for detecting cortically-located multiple sclerosis lesions or multiple sclerosis lesions afflicting the callosal radiation $(14,15)$. In addition, 3D constructive interference in steady-state images or axial T2weighted sequences were performed in patients with multiple sclerosis-related TN to screen for a vascular compression of the trigeminal nerve (9). 


\section{Lesion mapping}

Two experienced raters (KF and $\mathrm{KW}$ ) assessed the multiple sclerosis lesions on anonymized T2-weighted MRI sequences, the lesion boundaries were checked by a blinded third investigator (FS). Multiple sclerosis lesions were manually delineated on imaging scans using MRIcron software $(13,19)$. Lesion location was controlled for consistency on axial or 3D FLAIR scans, but delineated only on axial T2-weighted MRI scans. To ensure that no perivascular spaces were scored as multiple sclerosis lesions, lesions were only delineated if they were detectable on T2-weighted as well as on FLAIR scans as a hyperintense lesion. The MRI scan and the lesion shape were transferred into stereotaxic space using the normalization algorithm of SPM8 and the Clinical Toolbox for SPM8 (20-22). Because TN is a lateralized pain disorder, multiple sclerosis lesions of patients with clinically left-sided trigeminal neuralgia were flipped by the mid-sagittal plane $(23,24)$. Thus, using the MR-segment-normalize algorithm of the Clinical Toolbox, the MR images were transformed to the T1 template with resampled voxel size of $1 \times 1 \times 1 \mathrm{~mm}^{3}(20)$.

\section{Statistical analysis}

As a first step, we performed a subtraction analysis where the lesion overlap of multiple sclerosis patients without TN was subtracted from the lesion overlap of patients with multiple sclerosis-related TN $(23,24)$. As a second analysis, dichotomous overlap values of multiple sclerosis lesion sites identified in the VLSM analysis were correlated with the dichotomous behavioral variable, that is, whether or not the patient had trigeminal neuralgia using the Liebermeister test with 4000 permutations (24). Only voxels that were damaged in at least four individuals were included in this Liebermeister analysis. To control for multiple comparisons, we applied a family-wise error (FWE) correction of $p<0.05$.

Since increasing lesion volumes might be associated with an increased risk of afflicting brain areas that are involved in pain perception and endogenous pain modulation, we calculated volumes of multiple sclerosis lesions using nonparametric mapping (NPM) software implemented in the MRIcron software package (13). To determine damaged brain regions, affected voxels were overlaid on the Automated Anatomical Labeling (AAL) atlas (25). The peak coordinates of the involved regions are presented in Montreal Neurological Institute (MNI) space.

As a last step, a region-of-interest (ROI) based analysis was conducted, as ROIs served the areas for which the Liebermeister test showed a significant association with TN (the ipsilateral pontine brainstem ROI and the contralateral insular ROI). The binary variable was specified whether or not the patient had a lesion in the region of interest, the independent variable whether or not the patient had multiple sclerosis-related TN.

Demographic and clinical data were tested for normal distribution using the Shapiro-Wilk test and are presented as mean and standard deviation or median and interquartile ranges. Non-normally distributed data were compared using the Mann-Whitney U test or chi-square test, as appropriate. Two-tailed $p$-values were calculated, statistical significance was assumed for $p<0.05$. For statistical calculations, we used a commercially-available statistic program (SPSS 20.0; IBM, Armonk, NY).

\section{Results}

\section{Patient characteristics}

Of 12,210 patient records screened, 41 patients $(0.3 \%)$ fulfilled the inclusion criteria and had multiple sclerosis-related $\mathrm{TN}$, and were therefore eligible to be included in the VLSM analysis. Twenty one patients were positively screened but not included in the analysis; in 19 patients, no proper imaging could be obtained and in two patients imaging revealed a neurovascular contact. A total of 29 of the 41 patients $(71.0 \%)$ were women and $12(29.0 \%)$ were men. No cases of bilateral pain were reported. Clinical parameters and imaging characteristics of patients with multiple sclerosis-related $\mathrm{TN}$ and of the matched patients without TN are demonstrated in Table 1. A total of $60 \%$ were classified as relapsing-remitting multiple sclerosis, $33 \%$ as secondary progressive multiple sclerosis and $4 \%$ as primary progressive multiple sclerosis at the time of brain imaging. Patient age, disease duration, and EDSS score did not differ between patient groups with or without multiple sclerosis-related TN. The total lesion volume of patients with trigeminal neuralgia was significantly lower than the lesion volume of the control patients. Figure 1 shows the lesion distribution and lesion overlap of all patients. The highest lesion overlap, that is, the highest prevalence of individuals with lesions in a given voxel, was seen in the periventricular region, especially the parietal white matter, both insular and subinsular regions, the bilateral callosal radiation, and the periaqueductal midbrain gray.

\section{Voxel-based lesion symptom mapping}

Figure 2 shows the subtraction map of lesion overlaps in patients without TN subtracted from the lesion overlap of patients with TN. In short, lesioned voxels in the intrapontine trigeminal afferents and in the contralateral 
Table I. Demographic and clinical parameters of multiple sclerosis patients with and without trigeminal neuralgia.

\begin{tabular}{llll}
\hline & $\begin{array}{l}\text { Trigeminus neuralgia } \\
(\mathrm{n}=4 \mathrm{I})\end{array}$ & $\begin{array}{l}\text { No trigeminus } \\
\text { neuralgia }(\mathrm{n}=4 \mathrm{I})\end{array}$ & $P$ (two-tailed) \\
\hline Age, year; median (IQR) & $47(35-58)$ & $47.5(35-53.5)$ & $0.93 *$ \\
Disease duration, months; median (IQR) & $84(14.0-204)$ & $145(93.3-228.5)$ & $0.16^{*}$ \\
EDSS; median (IQR) & $3(2-6)$ & $5(3-7)$ & $0.87 *$ \\
Female/male & $29 / 12$ & $29 / 12$ & $1.00 \dagger$ \\
Lesion volume T2; voxels; median (IQR) & $24322(I 5393-7597 I)$ & $56720.5(32775-I I 5783)$ & $0.012 *$ \\
MS type (PPMS/RRMS/SPMS) & $2 / 24 / 15$ & $1 / 25 / 15$ & $0.92 *$ \\
\hline
\end{tabular}

IQR: interquartile range; EDSS: Expanded Disability Status Scale; PPMS: primary progressive multiple sclerosis; RRMS: relapsing-remitting multiple sclerosis; SPMS: secondary progressive multiple sclerosis.

*p-value derived from Mann-Whitney $U$ test.

$\dagger p$-value derived from Chi-square test.

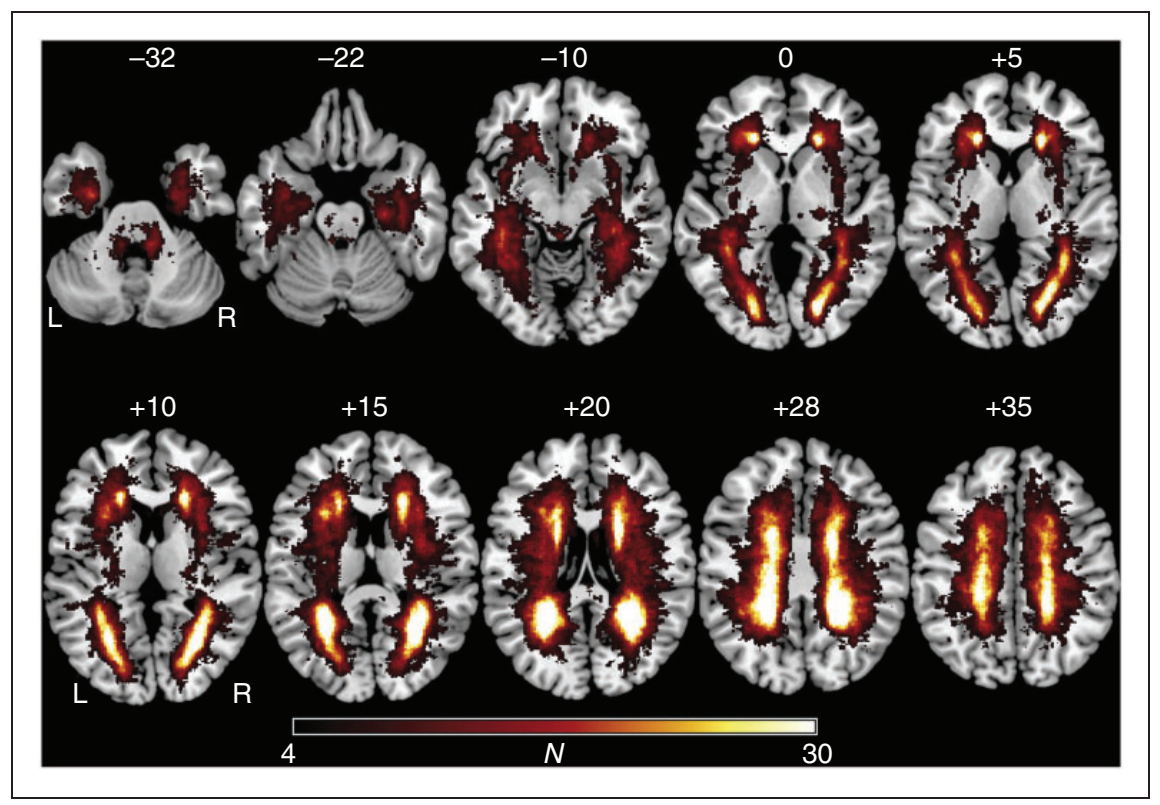

Figure I. Overlap and distribution of T2 lesions of all patients, thresholded to include only voxels that were lesioned in at least four individuals. The number of overlapping lesions is illustrated by different colors coding increasing frequencies from dark red to yellow. Supratentorial lesion pattern shows the highest overlay in subcortical and periventricular regions, especially in the parietal periventricular white matter. Infratentorially-summed lesion probabilty mapping indicates markable involvement of paramedian pontine regions.

L: left; $n$ : number of individuals with a lesion in a given voxel; R: right.

anterior insular regions as well as contralateral hippocampus were associated with multiple sclerosis-related TN. Table 2 illustrates brain areas in which lesioned voxels were associated with $\mathrm{TN}$ in the subtraction analysis, the corresponding voxel count and the peak coordinates in MNI space.

The non-parametric voxel-wise analysis using Liebermeister test statistics showed significant associations between trigeminal neuralgia and a total of 178 lesioned voxels. Of these, 15 voxels were located in the grey matter and 163 in the white matter. TN correlated significantly with multiple sclerosis lesions in ipsilateral pontine regions attributable to the trigeminal system, especially the region between the trigeminal root entry zone and the spinal trigeminal nucleus (thus, the intrapontine part of the trigeminal primary afferents). Furthermore, smaller sites of lesioned voxels remained associated with $\mathrm{TN}$ in the contralateral anterior insula (12 voxels; peak coordinates: $\mathrm{x}=-26 ; \mathrm{y}=24 ; \mathrm{z}=-3$ ) and the hippocampal region ( 2 voxels; peak coordinates: $\mathrm{x}=-32 ; \mathrm{y}=-24$; $\mathrm{z}=-7$ ) (Figure 3). 


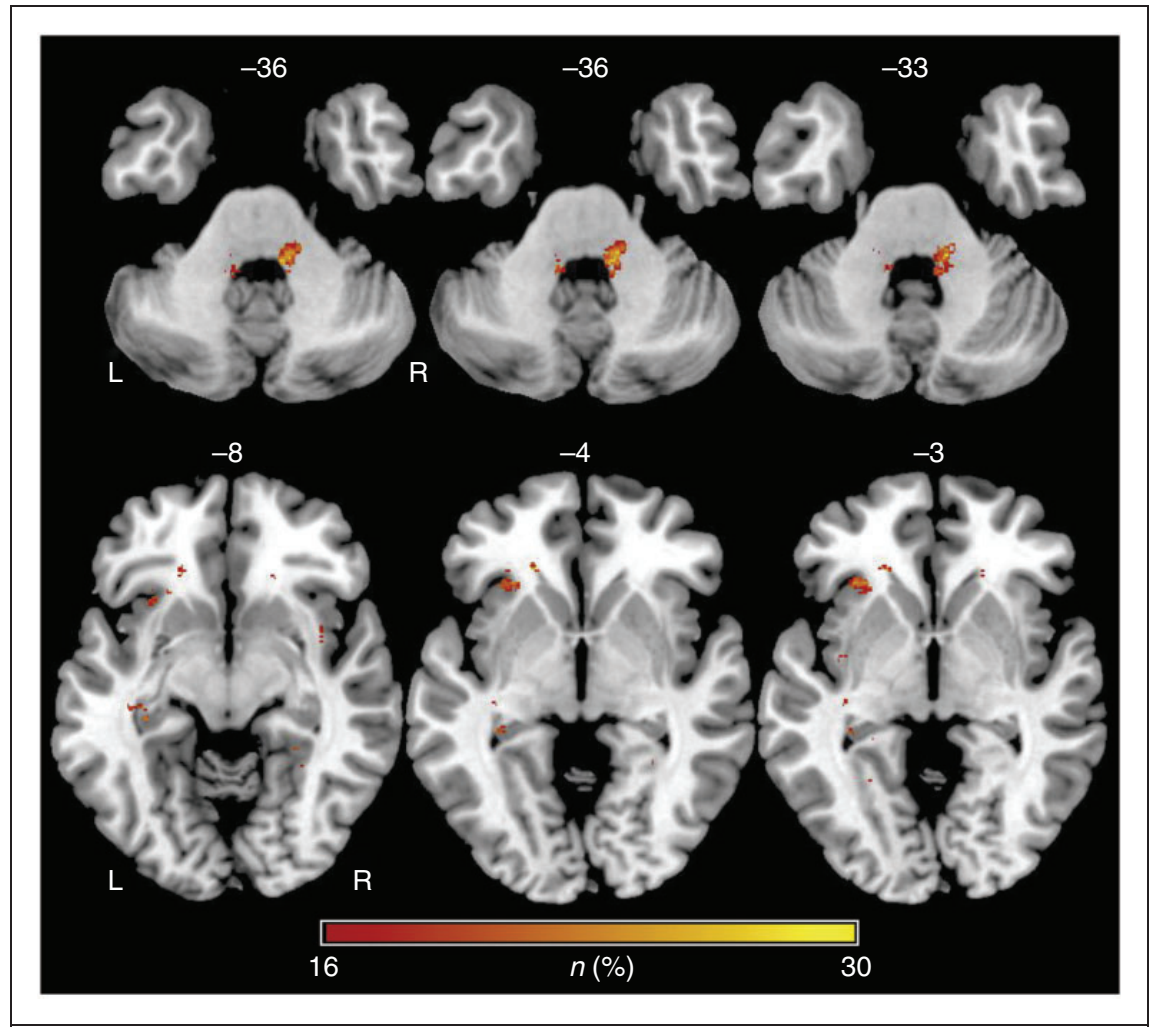

Figure 2. Subtraction map of lesion overlap of multiple sclerosis patients without TN subtracted from the lesion overlap of patients with TN. Lesioned voxels in the intrapontine trigeminal afferents but also in the contralateral insular region and contralateral hippocampus were associated with TN. The color bar indicates the number of patients in percent in whom a lesion in a given voxel remained associated with TN after subtraction. To reduce noise, only lesioned voxels that were affected in at least $16 \%$ of the individuals after subtraction are shown.

$\mathrm{L}$ : left; $\mathrm{n}$ : number of individuals in percent; R: right.

Table 2. Results from the voxel-wise subtraction analysis.

\begin{tabular}{lrrrr}
\hline Lesion site & \multicolumn{1}{c}{ Voxels } & \multicolumn{1}{c}{ x } & \multicolumn{1}{c}{ y } \\
\hline Insula (I) & 210 & -32 & 19 & -10 \\
Insula (r) & 14 & 38 & 8 & -9 \\
Hippocampus (I) & 112 & -33 & -30 & -8 \\
Hippocampus (r) & 52 & 27 & -35 & 0 \\
Calcarine (I) & 7 & -25 & -63 & 12 \\
Calcarine (r) & 57 & 16 & -87 & 3 \\
Fusiform (I) & 2 & -35 & -36 & -15 \\
Fusiform (r) & 26 & 28 & -42 & -10 \\
Caudate (I) & 4 & -15 & 1 & 18 \\
Caudate (r) & 16 & 21 & 23 & 16 \\
Cerebellum (I) & 13 & -10 & -44 & -38 \\
Cerebellum (r) & 11 & 10 & -43 & -35 \\
\hline
\end{tabular}

Brain areas according the areas defined in the Automated Anatomical Labeling (AAL) atlas in which lesioned voxels were associated with $T N$, as well as corresponding voxel counts and peak coordinates in MNI space are shown. I: left; r: right.

\section{Region of interest analysis}

Finally, we analyzed the relationship between multiple sclerosis lesions in (i) the brainstem and (ii) the anterior insula in the development of multiple sclerosis-related $\mathrm{TN}$ in a region of interest (ROI) analysis. As shown in Figure 4, a total of 19 patients $(46.3 \%)$ with TN and four patients $(9.8 \%)$ without $\mathrm{TN}$ had lesions both in the brainstem ROI and the insular ROI, five patients $(12.2 \%)$ with $\mathrm{TN}$ and two patients $(4.9 \%)$ without $\mathrm{TN}$ had lesions in the brainstem ROI but not the insular ROI, 13 patients $(31.8 \%)$ with $\mathrm{TN}$ and nine patients $(22.0 \%)$ without $\mathrm{TN}$ had lesions in the insular ROI but not the brainstem ROI, four patients $(9.8 \%)$ with $\mathrm{TN}$ and 26 patients $(63.4 \%)$ without $\mathrm{TN}$ had no lesion in the brainstem ROI and no lesion in the insular ROI.

\section{Discussion}

Trigeminal neuralgia is a severe, debilitating facial pain disorder that had a prevalence of $0.3 \%$ in our 


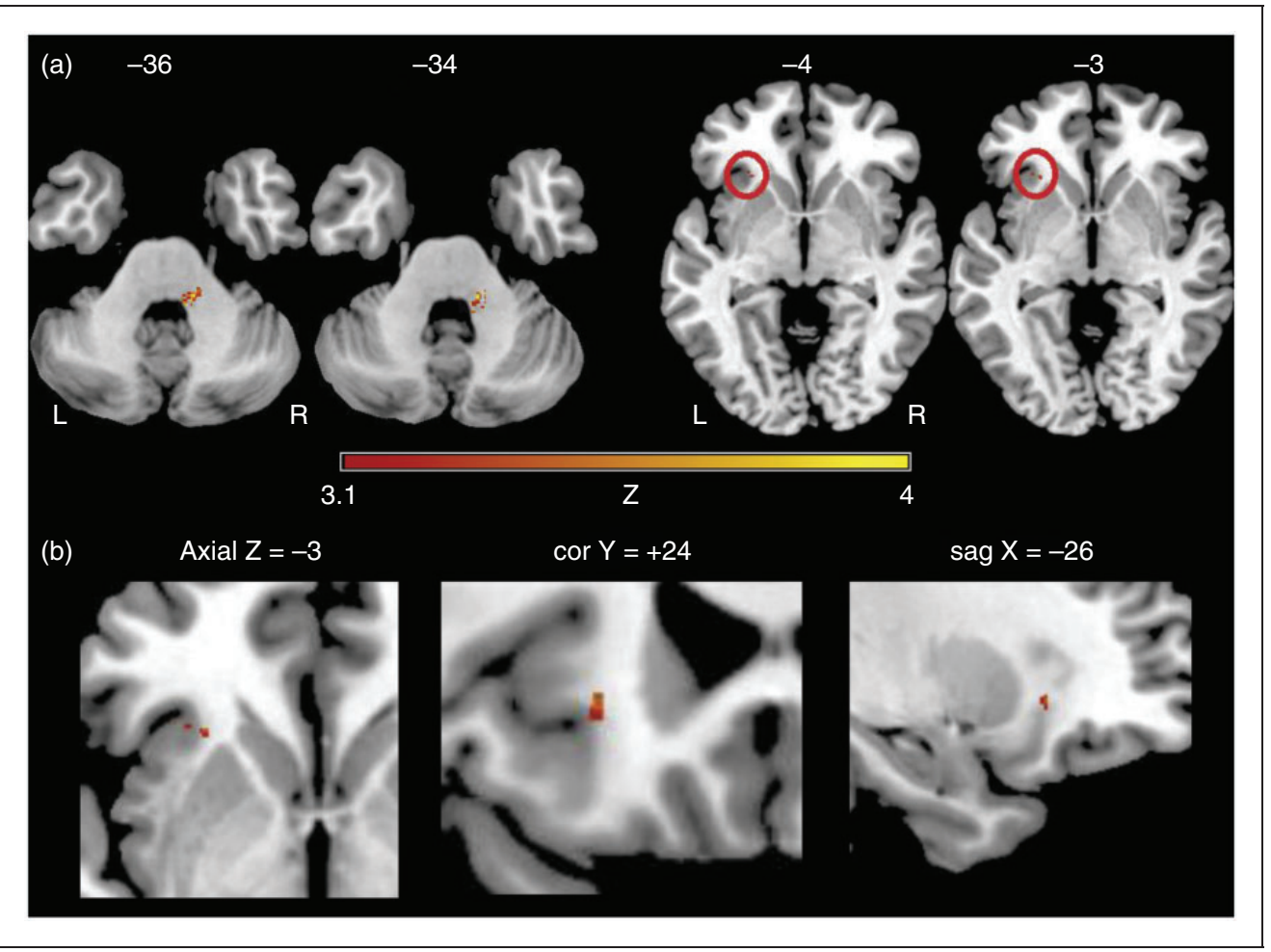

Figure 3. Results of the nonparametric Liebermeister test statistics. Lesioned voxels to be associated with TN are mainly found in the pons of the brainstem, in a region known to be part of the trigeminal nuclear system. Furthermore, smaller sites with significant lesioning associated with TN in multiple sclerosis were located in the insular region (a). In the lower row, we show the zoomed images in axial, coronar and sagittal planes illustrating lesioned voxels in the contralateral insular region (b). Only voxels that were damaged in at least four patients were included in the Liebermeister test analysis. A family wise error (FWE) correction of $p<0.05$ was applied. L: left; z: z-score; R: right.

retrospectively-analyzed multiple sclerosis patients. Our voxel-wise analysis indicates associations between multiple sclerosis-related $\mathrm{TN}$ and T2-hyperintense lesions most prominently in an ipsilateral brainstem area reaching from the pontine trigeminal root entry zone to the nucleus trigeminalis spinalis, but lesion clusters in the contralateral anterior insular region and hippocampus also seem to contribute to multiple sclerosis-related TN.

Previous imaging studies have investigated possible associations between $\mathrm{TN}$ in multiple sclerosis patients and brainstem lesions, but showed discrepant results $(2,6-10)$. Mills et al. showed that multiple sclerosis lesions in the brainstem affecting the trigeminal root entry zone were common but not necessarily associated with TN (10). In contrast, other imaging studies have shown a correlation between sensory facial symptoms or facial pain and pontine brainstem multiple sclerosis lesions or multiple sclerosis-related structural changes of the intrapontine course of the trigeminal nerve (2,6-9). Using multi-tensor tractography, Chen et al. recently showed different microstructural changes along the trigeminal nerve within the brainstem when they compared multiple sclerosis-related TN to the classic form of TN due to vascular compression (2).
In a multicenter study of 130 patients with multiple sclerosis, Cruccu et al. performed a voxel-based lesion analysis of brainstem lesions and found that multiple sclerosis lesions of the intrapontine trigeminal primary afferents were a strong predictor of TN (6). In another imaging study of 28 patients with multiple sclerosis and trigeminal sensory symptoms or TN, Truini et al. concluded that in addition to a pontine demyelinating multiple sclerosis lesion, a vascular compression of the trigeminal nerve similar to the classic form of $\mathrm{TN}$ might contribute to multiple sclerosis-related $\mathrm{TN}$ by means of a double-crush mechanism (9). However, in all these studies, multiple sclerosis lesions in other brain areas than the brainstem that contribute to integrating pain perception were not considered in the pathogenesis of multiple sclerosis-related TN $(2,6-10)$.

Therefore, in the present study, we performed a voxel-wise analysis of all T2-weighted multiple sclerosis lesions throughout the brain in a cohort of multiple sclerosis patients with TN (14). We determined the voxel-wise lesion pattern in our entire sample of multiple sclerosis patients with $\mathrm{TN}$ and compared their lesion overlap to the lesion overlap of a matched cohort of multiple sclerosis patients without TN. 


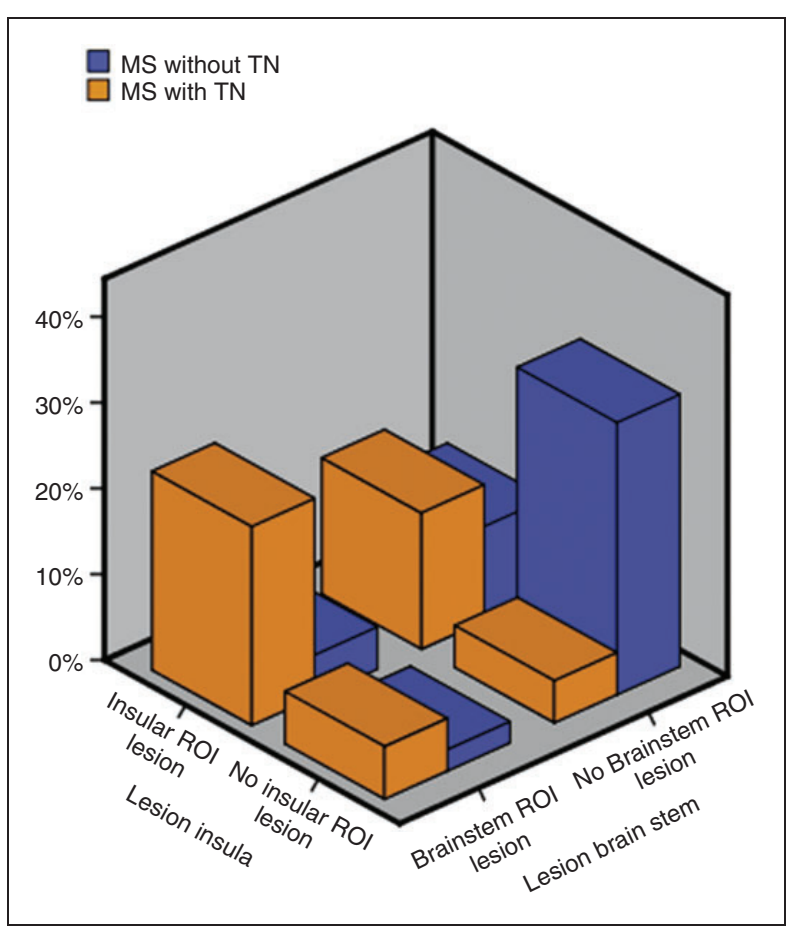

Figure 4. Overview of the lesion location of patients with and without trigeminal neuralgia in multiple sclerosis. The prevalence of multiple sclerosis patients with a lesion in the trigeminal root entry zone, in the contralateral insular region, as well as patients with lesions in both of these regions of interest are demonstrated.

$\%$ : percentage of the study population.

This rather specific approach towards identifying associations between circumscribed cerebral lesions and occurrence of TN confirms results of previous studies that pontine lesions of the intrapontine trigeminal primary afferents are associated with multiple sclerosisrelated TN (2,6-9). In our study, we observed a linear lesion pattern paramedian and intra-axial in the pons in continuity with the trigeminal nerve entering the pons. We propose that such a lesion pattern in the intrapontine trigeminal primary afferents is critical to develop multiple sclerosis-related TN (6-9). According to experimental studies, demyelinating lesions of the intrapontine trigeminal nerve fibers can increase susceptibility for ephaptic transmission $(11,26)$.

Surprisingly, our voxel-wise analysis showed that multiple sclerosis lesions in the contralateral insular region, most prominently in the anterior insula, were associated significantly with multiple sclerosis-related $\mathrm{TN}$. The insular cortex is a neural entity which contributes substantially to integrating painful nociceptive information (24,27-30). Pain and temperature sensation can be considered as interoceptive stimuli that are conveyed through common neural pathways $(27,29)$. Nociceptive painful information from the trigeminal nerve is conveyed to the brainstem and relayed to thalamic nuclei (27). Then interoceptive stimuli are further conveyed to the posterior insula, midinsula, and are finally integrated in the anterior insular cortex $(27,29,31)$. According to functional imaging studies in healthy individuals, there are differential pain processing effects of the anterior and posterior portion of the insular cortex $(27-29,32)$. The posterior insular cortex is particularly involved in encoding pain intensity, while the anterior insular cortex contributes more to the emotional or attentional components associated with painful sensation (27-29,32,33). In our study, we found an association between the occurrence of multiple sclerosis-related $\mathrm{TN}$ and lesions predominantly in the anterior insular cortex contralateral to the lesion in the pontine trigeminal root entry zone. Several previous studies linked lesions of central nervous system structures that are involved in pain processing to alterations of pain sensation or centrally-mediated pain $(23,24,27)$. It has been recognized that lesions of the thalamus can result in post-stroke pain syndromes (23). In a recent voxel-wise analysis of ischemic stroke patients, Seifert et al. linked stroke-associated headache to lesions of the insular cortex (24). Seifert et al. concluded that ischemic lesions of the insula may lead to alterations in a functional connectivity network of the pain matrix (24). The anterior insular cortex is involved in the cortical control of endogenous pain modulatory systems. A key brain region of the descending pain modulation pathway is the periaqueductal gray matter (PAG). The PAG receives input from anterior insular cortex as shown by animal studies (34). There is also evidence of restingstate functional MRI-based functional connectivity of the human PAG with the insular cortex (35). Disturbed or disrupted connectivity patterns between pain processing cortical areas and the pain modulatory system may contribute to the generation of episodic or chronic pain states $(36,37)$. Interestingly, a previous neuroimaging study has shown that the functional connectivity of the bilateral anterior insular cortex and the PAG as key areas of pain and pain modulatory networks relates to the susceptibility to pain (38). In this study, Ploner et al. found that a stronger functional connectivity of the anterior insular cortex with the PAG had antinociceptive effects on the perception of noxious stimuli. Therefore, an altered connectivity profile of these brain areas due to an insular multiple sclerosis lesion may increase susceptibility to pathologic discharges arising from lesions in the intrapontine trigeminal primary afferents.

Thus, we suggest that the intact anterior insular cortex normally increases thresholds to nociceptive input for - or stabilizes the nociceptive system against perceiving trigeminal neuralgic pain attacks. Thus, lesions of the insular cortex would thereby 
disinhibit neural structures of the pain-matrix, which in turn could lead to disinhibition of central nervous system structures like the nucleus trigeminalis spinalis, the anterior cingulate cortex or the posterior insular cortex that are involved in perceiving and processing painful input (27). The role of the hippocampus has been demonstrated during pain inhibition and its importance in pain perception and modulation, especially involving the cognitive-affective system, is widely accepted. The hippocampus has been shown to be linked to other brain regions, including the insula. Our findings are congruent with previous, as well neuroimaging results that an affection of the hippocampus may impair pain inhibition and mechanisms involved in the emergence of persistent pain (39).

\section{Limitations}

Yet our study has limitations that require a careful interpretation of the results. The prevalence of MSrelated TN was rather low, mainly resulting from underreporting in the retrospective setting, which could have influenced our results. Due to the retrospective study design, we did not have a standardized MRI protocol. Our intention was to exclude patients with a concomitant vascular contact of the trigeminal nerve by imaging to avoid bias or interference in our analysis. Because of the lack of suitable imaging, for example in high-resolution or adequate slice thickness in all patients, an underreporting of neurovascular contact is very likely. Besides, even if there is no evidence of vascular contact on imaging, a vessel-nerve contact cannot be ruled out completely. Patients were not contacted for the study, so it is possible that some patients in the study group experienced bilateral pain or patients in the control group experienced TN that was not accounted for in the analysis. Matching was performed for age, sex and EDSS. The implementation of further matching criteria, such as timing of imaging, would have been of additional value but was not possible due to the retrospective setting. In conclusion, we cannot rule out that disparities between the study and control groups, especially the lower lesion volume in the study group, could have affected the analysis. We assessed the multiple sclerosis lesions in our sample using T2-weighted MRI sequences. Hyperintense T2-lesions are unspecific and may represent lesions with possible regenerative potential. In contrast, T1-weighted hypointense lesions are more likely to represent persistent damage and axonal loss (40). Our hypothesis was that inflammatory MS lesions are associated with pain, but in fact we cannot exclude a different pathophysiology of the lesions, for example as a consequence of pain. Although preliminary analysis showed similar results, the examination of T1-lesion load was beyond the scope of the present study. A higher sample size might have produced different, maybe more clear results.

\section{Conclusion}

In conclusion, our data show associations between multiple sclerosis-related $\mathrm{TN}$ and intrapontine brainstem lesions affecting the trigeminal root entry zone. In addition, multiple sclerosis lesions of the contralateral insula and hippocampus may contribute to the pathogenesis of multiple sclerosis-related TN, possibly by attenuating thresholds for pain nociception.

\section{Article highlights}

- Brainstem lesions in the trigeminal root entry zone were associated with multiple sclerosis-related TN.

- Supratentorial multiple sclerosis lesions of the contralateral insula and hippocampus may contribute to multiple sclerosis-related TN.

\section{Declaration of conflicting interests}

The authors declared no potential conflicts of interest with respect to the research, authorship, and/or publication of this article.

\section{Funding}

The authors received no financial support for the research, authorship, and/or publication of this article.

\section{References}

1. Mohammad-Mohammadi A, Recinos PF, Lee JH, et al. Surgical outcomes of trigeminal neuralgia in patients with multiple sclerosis. Neurosurgery 2013; 73: 941-950.

2. Chen D, DeSouza D, Hayes D, et al. Diffusivity signatures characterize trigeminal neuralgia associated with multiple sclerosis. Mult Scler 2016; 22: 51-63.

3. Headache Classification Committee of the International Headache Society. The International Classification of Headache Disorders, 3rd edition (beta version). Cephalalgia 2013; 33: 629-808.

4. Vigeveno RM, Wiebenga OT, Wattjes MP, et al. Shifting imaging targets in multiple sclerosis: From inflammation to neurodegeneration. J Mag Res Imaging 2012; 36: $1-19$. 
5. Ge Y. Multiple sclerosis: The role of MR imaging. Am J Neuroradiol 2006; 27: 1165-1176.

6. Cruccu G, Biasiotta A, Di Rezze S, et al. Trigeminal neuralgia and pain related to multiple sclerosis. Pain 2009; 143: 186-191.

7. Gass A, Kitchen N, MacManus D, et al. Trigeminal neuralgia in patients with multiple sclerosis: Lesion localization with magnetic resonance imaging. Neurology 1997; 49: 1142-1144.

8. Nakashima I, Fujihara K, Kimpara T, et al. Linear pontine trigeminal root lesions in multiple sclerosis: Clinical and magnetic resonance imaging studies in 5 cases. Arch Neurol 2001; 58: 101-104.

9. Truini A, Prosperini L, Calistri V, et al. A dual concurrent mechanism explains trigeminal neuralgia in patients with multiple sclerosis. Neurology 2016; 86: 2094-2099.

10. Mills RJ, Young CA and Smith ET. Central trigeminal involvement in multiple sclerosis using high-resolution MRI at 3 T. Br J Radiol 2010; 83: 493-498.

11. Love $\mathrm{S}$ and Coakham H. Trigeminal neuralgia: Pathology and pathogenesis. Brain 2001; 124: 2347-2360.

12. Bates E, Wilson SM, Saygin AP, et al. Voxel-based lesionsymptom mapping. Nature Neurosci 2003; 6: 448-450.

13. Rorden $\mathrm{C}$, Karnath $\mathrm{H}$ and Bonilha L. Improving lesionsymptom mapping. J Cogn Neurosci 2007; 19: 1081-1088.

14. Winder K, Linker RA, Seifert F, et al. Neuroanatomic correlates of female sexual dysfunction in multiple sclerosis. Ann Neurol 2016; 80: 490-498.

15. Winder K, Seifert F, Koehn J, et al. Site and size of multiple sclerosis lesions predict enhanced or decreased female orgasmic function. J Neurol 2015; 262: 2731-2738.

16. Winder K, Seifert F, Köhrmann M, et al. Lesion mapping in stroke-related erectile dysfunction. Brain 2017; 140: 1706-1717.

17. Polman CH, Reingold SC, Banwell B, et al. Diagnostic criteria for multiple sclerosis: 2010 revisions to the McDonald criteria. Ann Neurol 2011; 69: 292-302.

18. Kurtzke J. Rating neurologic impairment in multiple sclerosis: An expanded disability status scale (EDSS). Neurology 1983; 11: 1444-1452.

19. McCausland Center for Brain Imaging. MRIcron software, http://www.mccauslandcenter.sc.edu/mricro/mricron (2007, accessed 04 June 2016).

20. Rorden C, Bonilha L, Fridriksson J, et al. Age-specific CT and MRI templates for spatial normalization. NeuroImage 2012; 61: 957-965.

21. Wellcome Department of Cognitive Neuroscience, University College London. Statistical Parametric Mapping (SPM), http://www.fil.ion.ucl.ac.uk (2012, accessed 04 June 2017).

22. McCausland Center for Brain Imaging. Clinical Toolbox for SPM8, http://www.nitrc.org/frs/?group_id=881 (2012, accessed 04 June 2017).

23. Sprenger T, Seifert CL, Valet M, et al. Assessing the risk of central post-stroke pain of thalamic origin by lesion mapping. Brain 2012; 135: 2536-2545.
24. Seifert CL, Schonbach EM, Magon S, et al. Headache in acute ischaemic stroke: A lesion mapping study. Brain 2016; 139: 217-226.

25. Tzourio-Mazoyer N, Landeau B, Papathanassiou D, et al. Automated anatomical labeling of activations in SPM using a macroscopic anatomical parcellation of the MNI MRI single-subject brain. NeuroImage 2002; 15: 273-289.

26. Love S, Gradidge T and Coakham HB. Trigeminal neuralgia due to multiple sclerosis: Ultrastructural findings in trigeminal rhizotomy specimens. Neuropath Appl Neurobiol 2001; 27: 238-244.

27. Craig A, Chen K, Bandy D, et al. Thermosensory activation of insular cortex. Nat Neurosci 2000; 3: 184-190.

28. Mazzola L, Isnard J, Peyron R, et al. Stimulation of the human cortex and the experience of pain: Wilder Penfield's observations revisited. Brain 2012; 135: 631-640.

29. Craig AD. How do you feel - now? The anterior insula and human awareness. Nat Rev Neurosci 2009; 10: 59-70.

30. Seifert F. Brain aging in female migraineurs: Differences in the insula. Pain 2015; 156: 1180-1181.

31. Critchley HD, Wiens S, Rotshtein P, et al. Neural systems supporting interoceptive awareness. Nat Neurosci 2004; 7: 189-195.

32. Bornhövd K, Quante M, Glauche V, et al. Painful stimuli evoke different stimulus-response functions in the amygdala, prefrontal, insula and somatosensory cortex: A single-trial fMRI study. Brain 2002; 125: 1326-1336.

33. Lorenz R, Gleich T, Kuehn S, et al. Subjective illusion of control modulates striatal reward anticipation in adolescence. Neuroimage 2015; 117: 250-257.

34. Leichnetz G, Spencer R and Hardy S. The prefrontal corticotectal projection in the monkey; an anterograde and retrograde horseradish peroxidase study. Neuroscience 1981; 6: 1023-1041.

35. Coulombe MA, Erpelding N, Kucyi A, et al. Intrinsic functional connectivity of periaqueductal gray subregions in humans. Hum Brain Mapp 2016; 37: 1514-1530.

36. Edwards R. Individual differences in endogenous pain modulation as a risk factor for chronic pain. Neurology 2005; 65: 437-443.

37. Seifert F, Kiefer G, DeCol R, et al. Differential endogenous pain modulation in complex-regional pain syndrome. Brain 2009; 132: 788-800.

38. Ploner M, Lee MC, Wiech K, et al. Prestimulus functional connectivity determines pain perception in humans. Proc Nat Acad Sci USA 2010; 107: 355-360.

39. Tian T, Guo L, Xu J, et al. Brain white matter plasticity and functional reorganization underlying the central pathogenesis of trigeminal neuralgia. Sci Rep 2016; 6: 36030.

40. Stefano N, Matthews P, Fu L, et al. Axonal damage correlates with disability in patients with relapsing-remitting multiple sclerosis. Brain 1998; 121: 1469-1477. 